[Vicino Oriente XX (2016), pp. 1-16]

\title{
UNA BROCCHETTA CON PROTOME D’ARIETE DALL'AREA SACRA DEL KOTHON A MOZIA
}

\author{
Federica Spagnoli - Sapienza Università di Roma
}

\begin{abstract}
During the $25^{\text {th }}$ and $26^{\text {th }}$ seasons of excavations carried out by Rome «La Sapienza» University Expedition to Motya (years 2015-2016) a ram's head jug was found in the Sacred Area of the Kothon (Area C South). From a typological point of view, this vessel can be considered a late outcome of the early Phoenician bronze trefoil jug, largely widespread over the western Mediterranean colonies, while its relevant symbolic value is represented by the ram's head, the animal traditionally consecrated to Baal, the deity worshipped in the Sacred Area.
\end{abstract}

Keywords: Motya; ram; Phoenician pottery; cult; temples

Durante le XXV e XXVI campagne di scavo a Mozia (2015-2016), è stata individuata e scavata, all'esterno del Temenos Circolare, poco a nord-est della Porta Sud (settore C Sud), una grande colmata (US.5108) che conteneva alcuni materiali, offerte rituali e oggetti utilizzati nel culto, provenienti dall'Area Sacra del Kothon. I materiali contenuti in questa gettata appartengono a epoche diverse e coprono l'intero arco cronologico di utilizzo dell'area, dall'inizio dell'VIII secolo al V secolo a.C. Questi furono deposti in momenti diversi, in concomitanza con le numerose risistemazioni architettoniche che interessarono questo settore dell'isola ${ }^{1}$. Tra i materiali rinvenuti nella colmata è una brocchetta dipinta (MC.15.5108/1) di raffinata fattura, che reca una protome di ariete alla sommità del collo, al di sotto dell'imboccatura. L'interesse destato da questo reperto ha una duplice valenza, tipologica ed ideologica. Esso infatti può essere considerato un esito recenziore, punico, di una tipologia di brocche fenicie molto antica, le brocche biconiche a orlo trilobato, ma è anche un oggetto dalla forte valenza simbolica relativa agli aspetti religiosi e culturali rappresentati nell'area.

\section{IL CONTESTO DI RINVENIMENTO: I MATERIALI DALLA COLMATA US.5108}

I materiali ceramici rinvenuti all'interno della colmata US.5108 coprono un arco cronologico che va all'inizio dell'occupazione fenicia dell'area (inizio dell'VIII secolo a.C.) al V secolo a.C. La colmata è con ogni probabilità immediatamente successiva alla distruzione finale del 397/6 a.C. e rappresenta i lavori di risistemazione dell'area per l'apprestamento del Santuario C32 ${ }^{2}$. Durante questi lavori, il pareggiamento e la rimozione dei resti degli edifici più antichi portarono ad intaccare gli strati anteriori sottostanti fino a raggiungere anche i livelli dell' VIII e VII secolo a.C. È stato dunque possibile individuare tre gruppi distinti di ceramica cronologicamente omogenei che riflettono a loro volta, seppur parzialmente, i diversi periodi di vita della città in questo settore. La brocchetta rappresenta il reperto più recente finito nella colmata e deve essere stata in uso nel vicino Tempio C2 al momento della distruzione finale della città da parte di Dionigi di Siracusa.

1 Nigro 2013a, 39, tab. 1; Nigro 2015, 226, tab. 1.

2 Nigro ed. 2005, 60 e segg.

ISSN 0393-0300

e-ISSN 2532-5159 
1.1. Il nucleo più antico: la ceramica Red Slip e indigena d'impasto di VIII secolo a.C. (Motya IVA-B, Fasi 9-8, 800-675 a.C.)

Il nucleo più antico è rappresentato dai materiali pertinenti alle prime fasi di utilizzo dell'Edificio C8 (Motya IV, 800-675 a.C.) rimossi al momento della ricostruzione dell'edificio avvenuta nel VII secolo a.C. (Fase 7, 675-550 a.C.) ${ }^{3}$. È il lotto di materiali più numeroso tra quelli analizzati nel presente studio, in cui si distingue un nucleo più antico databile entro la prima metà dell'VIII secolo a.C. (Motya IVA, 800-750 a.C.) (fig. 1): due coppe di impasto (MC.16.5108/9 e MC.16.5108/14) ${ }^{4}$, due teglie (MC.15.5108/35, MC.15.5108/31) ${ }^{5}$ e un orciolo (MC.15.5108/33) ${ }^{6}$ e, tra la ceramica fenicia, un'anfora con decorazione dipinta in rosso (MC.15.5108/13), e un'anfora levantina (MC.16.5108/11). A questa prima fase sono attribuibili anche una lama di ossidiana (MC.16.39) e una macina in basalto (MC.16.30). Tra i materiali ascrivibili alla seconda metà dell'VIII-primo quarto del VII secolo a.C. (Motya IVB, 750-675 a.C.) si annoverano una coppa carenata dipinta $\left(\right.$ MC.16.5108/8) ${ }^{7}$ e due bicchieri (MC.15.5108/3, MC.15.5108/23) in Red Slip di produzione locale, un'anfora da dispensa dipinta (MC.15.5108/24) e un aryballos Protocorinzio dipinto con una teoria di cani correnti (MC.16.5108/16) ${ }^{8}$.

\subsection{I materiali di VII secolo a.C. (Motya VA, Fase 7, 675-625 a.C.)}

Quando l'Edificio C8 venne rasato in concomitanza dell'erezione del Temenos Circolare (Motya VB-VIA, Fasi 6-5, 625-550/550-520 a.C.) ${ }^{9}$, i materiali in uso nell'ultima fase di vita dell'edificio (Motya VA, Fase 7, 675-625 a.C.), vennero raccolti e gettati nella colmata US.5108. La ceramica ascrivibile a questa fase (fig. 2) comprende due piatti fenici, uno con decorazione bicroma sulla tesa (MC.16.5108/6) e uno dipinto (MC.15.5108/15), due olle da fuoco (MC.15.5108/18 e MC.16.5108/12) ${ }^{10}$ e una brocca (MC.15.5108/18). Tra la ceramica di importazione si annovera un'anfora attica di tipo SOS attribuibile alla prima metà del VI secolo a.C.

\subsection{I materiali di V secolo a.C. (Fase 4, Motya VIII, 470-397 a.C.)}

La ceramica appartenente a questo gruppo proviene con ogni probabilità da uno degli edifici sacri presenti nell'area sacra. Per le categorie di oggetti rinvenuti e per le tipologie ceramiche rappresentate è verosimile supporre che questi materiali costituissero gli arredi cultuali e le offerte votive del Tempio $\mathrm{C} 2{ }^{11}$, interrati nella colmata subito dopo la conquista siracusana durante la quale l'area venne rasa al suolo. A ridosso dell'invasione dionigiana, infatti, le strutture sacre, come pozzi, stele e betili, vennero defunzionalizzate o smontate ${ }^{12}$,

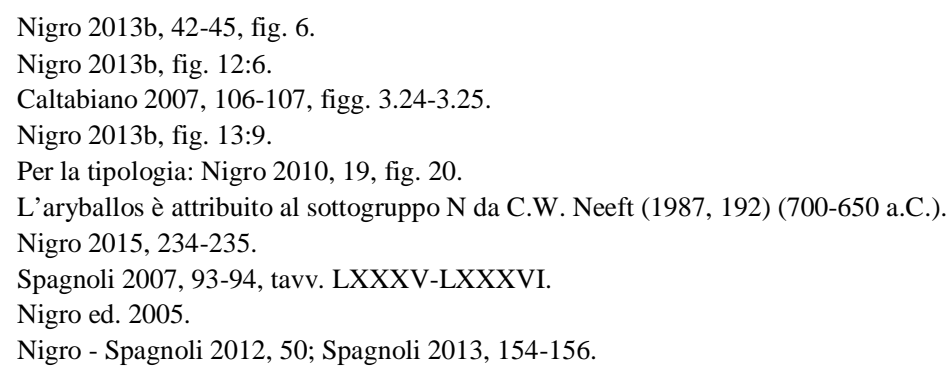


e gli oggetti sacri portati fuori dall'edificio sacro e interrati ${ }^{13}$. Gli oggetti e la ceramica rinvenuti all'interno della colmata, infatti, sono quelli che si ritrovano tradizionalmente tra le offerte votive dei depositi del Tempio della Fase $4{ }^{14}$, mentre altri, come le piastre a rilievo (pinakes) e le colonnine fittili, costituivano probabilmente gli arredi mobili dell'edificio di V secolo a.C.

Gli oggetti rinvenuti sono quelli caratteristici dei depositi votivi del Tempio del Kothon nella fase: punte di freccia e di giavellotto (MC.13.109, MC.13.134, MC.15.102), oscilla e pesi (MC.16.2, MC.15.87, MC.13.123), pepite (MC.15.89), chiodi ripiegati (MC.13.115, MC.13.117, MC.15.85, MC.15.115), oggetti di bronzo (MC.16.194, occhiello, MC.15.104, lamina), coralli e oggetti in osso (MC.15.81, MC.15.96, MC.15.105), scarti di lavorazione di metalli (MC.16.166, scoria di bronzo) ${ }^{15}$, e alcuni arredi fittili (MC.13.110, MC.13.139) ${ }^{16}$. Tra la ceramica sono compresi coppe e fondi di skyphoi a vernice nera ritagliati $^{17}$ (MC.16.5108/7), coppette a vernice nera e acrome (MC.16.5108/1, MC.15.5108/2, 8, 11, 22) ${ }^{18}$ e, infine, la brocchetta con protome d'ariete oggetto di questo studio (MC.15.5108/1).

La colmata fu poi sigillata dalle macerie dell'area sacra ${ }^{19}$, livellate in occasione della sistemazione dell'area in luogo di culto a cielo aperto (Santuario C3) ${ }^{20}$.

\section{LA BROCCHETTA CON PROTOME D’ARIETE MC.15.5108/1}

La brocchetta MC.15.5108/1 costituisce un rinvenimento di grande valore sia dal punto di vista della manifattura sia per la possibile valenza simbolica del soggetto rappresentato, soprattutto se considerato nel suo contesto originario di provenienza. Si tratta, infatti, di un oggetto che trova confronti sia nell'area fenicia orientale sia occidentale e principalmente, ma non solo, in contesti cultuali.

\subsection{Morfologia del vaso e confronti tipologici}

La brocchetta con protome d'ariete MC.15.5108/1 è conservata in quasi tutta l'altezza originaria: la parte preservata è alta $17 \mathrm{~cm}$, su un'altezza complessiva stimata di circa 20 $\mathrm{cm}$, mentre la porzione inferiore del corpo e la base sono andate perdute (fig. 3). La brocchetta presenta un orlo rettilineo indistinto molto stretto $(1 \mathrm{~cm})^{21}$, innestato alla sommità dell'applique conformata a testa di ariete. Il collo della brocchetta scende leggermente rastremato, l'ansa a nastro parte immediatamente sotto l'orlo e si attacca sulla spalla. La forma di quest'ultima e della pancia del vaso sono leggermente asimmetriche, tanto da far supporre che il vaso dovesse avere una forma irregolare schiacciata nel punto di massimo diametro e lievemente biconica. Il collo e l'orlo, inoltre, sembrano incurvati o

3 Alcuni dei reperti attribuiti alla US.4448 scavata nel 2013 e nel 2015 sono pertinenti a questa gettata.

14 Nigro ed. 2005, 73-85; Nigro 2009, 706-708; Nigro - Spagnoli 2012.

15 Nigro ed. 2005, 74, tav. XLIII.

16 Nigro ed. 2005, 110, 116.

7 Nigro - Spagnoli 2012, 40, con bibliografia precedente.

18 Sparkes - Talcott 1970, 102, fig. 5: 48 (425 a.C.).

19 Nigro ed. 2005, 45-48, nota 51.

20 Nigro ed. 2004, 45 e segg.

21 La brocchetta è stata restaurata dal M. Salvatore Tricoli, che sentitamente ringrazio. 
inclinati leggermente verso l'ansa, come per bilanciare l'asimmetricità del corpo e permettere una visione di tre quarti, con una inclinazione di 45 gradi, della protome.

Dal punto di vista tipologico, la brocca deriva da una forma di tradizione fenicia, la brocca piriforme con orlo trilobato ${ }^{22}$, della quale è una rielaborazione tarda con alcune differenze morfologiche e stilistiche. Lo stacco alla base del collo evidenziato da una risega e da un cordolo all'innesto della parte inferiore del vaso è molto poco marcato, ma è concettualmente analogo a quello delle brocche piriformi trilobate in metallo e in ceramica di VIII e VII secolo a.C. ${ }^{23}$. Un'altra differenza tra la brocchetta con protome d'ariete e le brocche piriformi fenicie più antiche è data dalla forma dell'ansa, che nella prima è a nastro, mentre nelle seconde (specialmente negli esemplari più antichi) è a doppio bastoncello $^{24}$. Se la posizione della protome d'ariete subito sotto l'orlo ricalca la composizione delle brocche arcaiche con appliques, il fatto che il muso dell'ariete non presenti i fori per versare appare come una differenza sostanziale rispetto ai modelli fenici: l'unica apertura presente nel vaso, così come è conservato, è l'imboccatura superiore, segnata da un orletto dalla forma cilindrica che non trova confronti negli esemplari tipologicamente affini più antichi.

\subsection{Cronologia}

La cura delle superfici e la precisione della modellazione plastica iscrivono questo reperto tra gli oggetti di maggior pregio rinvenuti nell’ Area Sacra del Kothon.

Il vaso è ricoperto da una ingubbiatura bianco-beige ${ }^{25}$ sulla quale è una decorazione dipinta in rosso scuro ${ }^{26}$, secondo lo stile della ceramica dipinta moziese nei periodi Motya VI e Motya VII (VI-V secolo a.C.) ${ }^{27}$. In particolare, la somiglianza dell'impasto, del rivestimento superficiale e della decorazione dipinta tra questa e un'altra brocchetta proveniente dal Tempio di Astarte (MC.14.4362/2) all'interno della stessa area sacra, porta a pensare che possa trattarsi di produzioni cronologicamente molto vicine ascrivibili alla metà del V secolo a.C. ${ }^{28}$.

\subsection{Decorazione dipinta e decorazione plastica}

La decorazione pittorica è articolata in modo da mettere in risalto la tettonica del vaso: tre linee orizzontali sono poste sul punto più ampio del corpo, e sul collo sono tratti verticali che, partendo dalla spalla, arrivano all'attacco della protome. La stessa protome presenta delle parti dipinte: le corna sono dipinte in rosso, così come la bocca, le orecchie, il profilo del muso e le narici, e la parte sommitale della testa, laddove si innesta l'imboccatura, sono evidenziati dalla pittura (fig. 4). L'ansa a nastro è decorata con linee orizzontali.

22 Grau-Zimmermann 1978, figg. 1-6.

23 Culican 1982, 68, 75, fig. 9a; Nuñez 2004, 320, fig. 180, Achziv ZR XXXVI, note 213, 354, seconda metà VIII-inizi VII secolo a.C.

24 Taloni 2012a, 375.

25 Munsell Soil Color Chart 2013: 10YR8/3, Very Pale Brown.

26 Munsell Soil Color Chart 2013: 2.5YR4/6, Red.

27 Spagnoli 2014, 114-115.

28 Spagnoli 2014, 117, fig. 1. 
La modellazione plastica della protome è molto accurata, con una particolare attenzione per il dettaglio anatomico. Le corna ricurve sono rese da due cercini attorcigliati che arrivano quasi all'altezza della bocca, leggermente aperta. Gli occhi sono ricavati da due fessure intagliate, all'interno delle quali è stata applicata una piccola sfera, le narici e la bocca sono rese da incisioni poco profonde. Le orecchie tonde, incorniciate dalle corna, sono meticolosamente rese nel dettaglio e sono dipinte. Alla sommità della testa si innesta l'imboccatura del vaso. Come si è accennato in precedenza, la protome non presenta fori per versare, a differenza di altri esemplari più antichi in cui il muso dell'ariete di solito funge da versatoio.

Per quanto riguarda l'aspetto iconografico, i confronti più vicini provengono da Mozia stessa, in particolare dall'area del Cappiddazzu (fig. 5) ${ }^{29}$. Lo stile della raffigurazione trova dei confronti puntuali anche nei coevi rhyta greci: la resa delle corna e del muso dell'animale, infatti, ricorda in particolare le produzioni attiche a figure rosse della prima metà del V secolo a.C. ${ }^{30}$

La presenza, invece, dell'applique plastica conformata a testa di ariete posta presso l'imboccatura del vaso, solitamente, ma non nel nostro caso, utilizzata come beccuccio versatoio, è un elemento comune nelle produzioni orientali di epoche precedenti. Le applicazioni plastiche sulle brocche hanno una lunghissima tradizione risalente nel Levante, a Cipro e nel Vicino Oriente, al periodo Calcolitico ${ }^{31}$. In quest'epoca, e successivamente anche nel Bronzo Antico e Medio ${ }^{32}$, i soggetti rappresentati sono, oltre all'ariete, anche serpenti, tori e capridi in generale ${ }^{33}$. Questi compaiono soprattutto su vasi cultuali utilizzati in ambito funerario e sacro, e sono connessi ai riti pastorali di libagione finalizzati a propiziare la fertilità e la fecondità delle mandrie ${ }^{34}$. In area Egea tra la fine del terzo e il secondo millennio a.C. appliques conformate a testa di ariete compaiono sulle coppe rituali utilizzate per libagioni, le cosiddette sauceboats ${ }^{35}$. Anche a Cipro recipienti e statuette criomorfe hanno lunga tradizione, e l'ariete, al pari del toro, viene spesso riprodotto come applique plastica nei vasi in Red Burnished Ware con funzione cultuale o funeraria ${ }^{36}$. Nella ceramica cipro-fenicia di X-IX secolo a.C., decorazioni plastiche erano frequenti nelle produzioni dipinte: si ricorda, a titolo esemplificativo, una brocchetta Black on Red con protome d'ariete con bocca passante, applicata alla parte terminale del beccuccio versatoio sulla spalla, conservata nel Pierides Foundation Museum di Larnaca. Tra la fine del IX e il VII secolo a.C. i Fenici sono i vettori della diffusione di questo tipo di vasi con appliques anche in Occidente ${ }^{37}$ : brocche piriformi trilobate metalliche e in ceramica con protomi di ariete, cervo, leone, e anche di animali fantastici quali il grifone, sono frequenti dalla

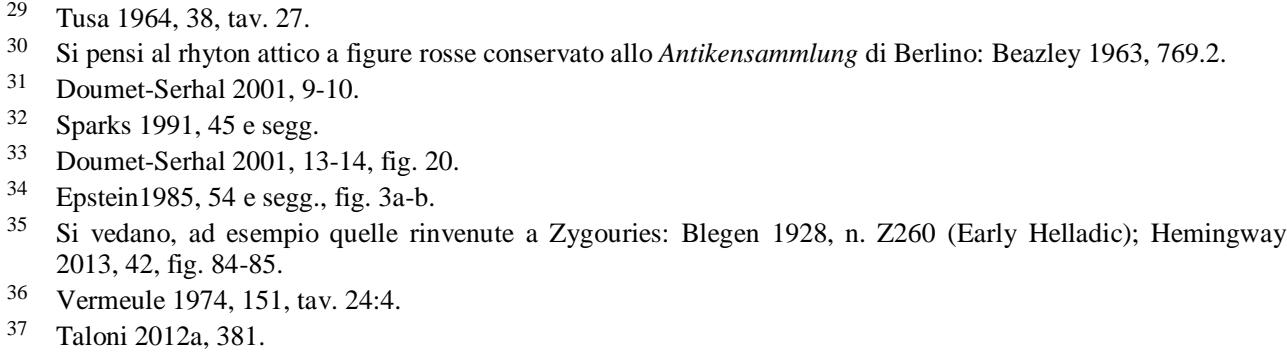


penisola iberica all'area tirrenica e centro mediterranea principalmente in contesti funerari ${ }^{38}$. In Egitto brocche in bronzo, ma anche in alabastro, con la sommità conformata a testa di ariete, sono state rinvenute in alcune tombe reali nel VII secolo a.C. Si tratta di oggetti importati dalla costa levantina o prodotti localmente da artigiani fenici ${ }^{39}$.

Nello stesso periodo la protome d'ariete compare in Occidente anche applicata su un altro tipo di vaso di uso prettamente funerario, il kernos circolare, sempre con il muso forato per versare ${ }^{40}$. Attestati nel Vicino Oriente fin dal Tardo Bronzo ${ }^{41}$, i kernoi con protome d'ariete sono presenti a Cipro nel Tardo Cipriota I (1650-1450 a.C.), mentre all'inizio dell'Età del Ferro si diffondono anche in Fenicia ${ }^{42}$ e, successivamente, dalla fine dell'VIII secolo a.C., anche in Occidente, in area tirrenica ${ }^{43}$, magno-greca ${ }^{44}$ e fenicia coloniale $^{45}$. Uno degli esemplari occidentali più antichi di kernos con protome d'ariete è stato rinvenuto proprio a Mozia nella Tomba 29 della Necropoli Arcaica (fig. 6) ${ }^{46}$. L'esemplare moziese presenta una complessa decorazione con linee in pittura nera su ingubbiatura rossa. L'applique plastica a forma di testa di ariete è incorniciata da linee di pittura, e ha il muso prominente, gli occhi e le narici incisi, la bocca con foro passante, le corna semicircolari prive di estremità, le orecchie lunghe e appoggiate orizzontalmente sulle corna. I vasi a calice che si collocano lateralmente rispetto alla applique, che costituisce il beccuccio versatoio del kernos, sono uniti tra di loro attraverso gli orli ${ }^{47}$. La cronologia del vaso, inizialmente attribuita alla fine del VII secolo a.C., è stata successivamente rialzata, prima su base tipologica ${ }^{48}$ e poi anche in relazione ai dati di contesto, alla prima metà dello stesso secolo.

38 Grau-Zimmermann 1978, tav. 1, figg. 1:5, 8. Brocche o oinochoai con bocca configurata, sia in metallo sia in ceramica, che presentano protomi leonine: brocca in bronzo, Madrid, Museo Lázaro Galdiano (Jiménez Ávila 2002, 387, tavv. V-VI, n. 10); oinochoe in bucchero, Bruxelles, Musée Royaux d'Art et d'Histoire (Gran Aymerich 1983, 81, fig. 2; Bruxelles A777). Brocche che presentano protomi di cervo: brocca in bronzo, Collezione Calzadilla - Museo Archeologico provinciale di Badajoz; brocca in bronzo, tomba T.18, necropoli di La Joya a Huelva, Museo Provincial (Jiménez Ávila 2002, 388, tavv. VII-VIII, nn. 11-12). Brocche con protomi di grifo: brocca in bronzo, Bruxelles, Musée Royaux d'Art et d'Histoire (Paris 2007, 350, cat. 190).

40 L'iconografia dell'ariete è presente nel mondo punico anche in altre classi di materiali, come l'astuccio porta amuleti dalla necropoli di Cartagine, datato tra il VII e il VI secolo a.C. (Lancel 1992, 85-86, fig. 45:a).

41 Tra questi si citano i kernoi da Tell Bazi (Siria) di XIII secolo a.C. e da Tell Judeide di XI secolo a.C. (Bignasca 2000, 113).

42 Sulla costa Levantina l'esemplare più arcaico è datato all’VIII secolo a.C. ed è stato rinvenuto a Ashdod (Dothan - Porath 1982, 26, 32, 41, figg. 11:9, 18:1, 28:1-2)

43 Si citano a titolo esemplificativo i kernoi da Populonia (Minto 1934, 371, fig. 26.1) (VI secolo a.C.); da Vetulonia (Poggio alla Guardia) (Camporeale 1962, 4, tav. 1:a, b) (VII secolo a.C.); da Tarquinia (Monterozzi) (Hencken 1968, 387, fig. 377) (VII sec. a.C.); da Francavilla Marittima (Stoop 1976, tavv. 5556).

44 Bignasca 2000, 192.

45 Bartoloni 1992, 129-130.

46 Tusa 1972, 70-71, LII-LIII, XCVI.

47 Un altro esemplare, simile ma di un cinquantennio posteriore, è stato rinvenuto in una tomba di Bithia (Bartoloni 1992, 129-130).

48 Bartoloni 1992, 131. 


\section{CONCLUSIONI: L’ARIETE E IL CULTO DI BAAL CTONIO NELL’AREA SACRA DEL KOTHON}

La raffigurazione dell'ariete sulla brocchetta MC.15.5108/1, un vaso di culto che probabilmente era originariamente collocato all'interno del Tempio di Baal, è un chiaro riferimento a una divinità legata alla fertilità della terra, delle greggi e degli uomini, nonché alla ricchezza del sottosuolo. Nella tradizione greca (pur se trasmessa da mitografi tardi, che fanno riferimento anche alla mitologia egiziana), l'ariete è un animale legato anche alle sorgenti di acqua dolce ${ }^{49}$. L'intera Area Sacra del Kothon sorge in funzione delle numerose sorgenti di acqua dolce di cui la zona meridionale di Mozia in particolare è molto ricca ${ }^{50}$. Anche l'associazione frequente, in ambito fenicio come in quello greco ${ }^{51}$, tra l'ariete e i vasi cultuali utilizzati per versare, come brocchette, askoi, anforette e kernoi, questi ultimi rinvenuti, in ambito fenicio e punico, principalmente nelle sepolture, costituisce un ulteriore richiamo al tipo di riti officiati nell'area sacra, riti che prevedevano la libagione in orifizi che mettevano in contatto l'officiante/offerente con il mondo sotterraneo ${ }^{52}$. L'accostamento a Baal, divinità titolare del tempio maggiore dell'Area Sacra, come testimoniato anche dall'iscrizione sull'aryballos laconico rinvenuto nei pressi del Tempio ${ }^{53}$, appare quindi quasi inevitabile: l'ariete sarebbe l'animale simbolo del Baal ctonio, legato alle acque sotterranee, alla fusione dei metalli, e rappresenterebbe la potenza del dio come fecondatore delle greggi e degli uomini. Se dal punto di vista iconografico i confronti più prossimi per la protome sono da ricercarsi in ambito greco, per quel che riguarda l'origine e il significato della rappresentazione si deve guardare alla più antica tradizione religiosa orientale, e in particolare a quella egiziana. Sembra infatti possibile poter escludere un accostamento alla divinità levantina Baal Hammon ${ }^{54}$, come invece avviene per le rappresentazioni cipriote, ad esempio quelle provenienti dal santuario rurale di Menikò ${ }^{55}$. Nel caso della brocchetta moziese, infatti, il legame con Baal Hammon non è supportato né da elementi di contesto (tra i materiali della colmata mancano completamente, ad esempio, i caratteristici bruciaprofumi o incensieri, tipico attributo della divinità), né da caratteristiche intrinseche

Bignasca 2000, 177.

50 Nigro - Spagnoli 2012, 2-6.

51 In ambiente greco-orientale le raffigurazioni di ariete su askoi sono comuni nel periodo Geometrico. Si vedano, come esempio, un askos conservato allo Oxford Ashmolean Museum (CVA Ashmolean Museum 2, 75 tav. 1:4) e un altro simile conservato al Copenhagen National Museum (CVA Copenhagen 2, 61, tav. 81:8), entrambi provenienti da Rodi.

52 Nigro 2009, 705-706.

53 Guizzi 2012.

54 La più antica rappresentazione di Baal Hammon nel mondo fenicio e punico è costituita da un anello rinvenuto nella necropoli di Dermech da P. Glauker, datato al VI secolo a.C. L'iconografia è simile a quella del Baal in trono nella barca solare che appare sui sigilli, con in alto il disco solare che richiama il legame con la barca di Osiride e con la concezione del viaggio dell'astro nel mondo dei morti. Il personaggio è vecchio e barbuto, una tiara appuntita sulla testa, seduto su un trono ad alto schienale, fiancheggiato da sfingi (simile ai troni c.d. di Astarte). Nella mano ha un oggetto non bene identificato (spiga di grano?). Davanti al dio c'è un braciere e in basso un animale marino, forse un serpente a due teste (o un ippocampo), tra l'animale e la barca un fiore a stelo lungo che rappresenta il potere universale e cosmico della divinità rappresentata (Xella 1991, 114-115, tav. VII:1).

55 Karageorghis 1977, 39-41. 
all'oggetto che ne possano indirizzare l'attribuzione ${ }^{56}$. La valenza simbolica dell'ariete nel contesto dell'Area Sacra del Kothon sembra, invece, più vicina una delle divinità principali del pantheon egiziano del periodo protodinastico: Khnum, raffigurato con la testa d'ariete, divinità creatrice e protettrice delle sorgenti del Nilo e della potenza creatrice delle inondazioni ${ }^{57}$, le cui prerogative vennero gradualmente assorbite da Amon ${ }^{58}$.

Ancora una volta, dunque i reperti provenienti dall'area sacra riconducono a un ambito

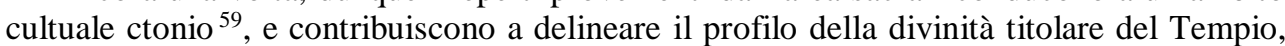
un Baal polivalente legato alla terra e alla potenza fecondatrice della natura ${ }^{60}$, ma anche alle acque sotterranee, alla navigazione e alle stelle che avevano guidato le navi dei Fenici fino a Mozia quando questi, come Europa, figlia di Agenone re di Tiro e di Sidone, sul dorso di Zeus (il Baal fenicio) nelle sembianze di un toro, attraversarono il Mediterraneo per stabilirsi in un Occidente lontano, contribuendo alla formazione della nostra civiltà mediterranea.

\section{BIBLIOGRAFIA}

BARTOLONI, P.

1992 Recipienti rituali fenici e punici dalla Sardegna: Rivista di Studi Fenici 20 (1992), pp. 123-142.

BEAZLEY, J.D.

$1963 \quad$ Attic Red-Figure Vase-Painters, Oxford $1963^{2}$.

56 L'accostamento tra Baal Hammon e l'ariete è rappresentato, ma in un contesto posteriore, nelle stele del tofet di Sousse (Hadrumetum) e El-Hofra (Costantina) (D’Andrea 2014; Spagnoli 2015, 222), nonché nelle stele tarde di III secolo a.C. da Sulcis (I Fenici, nn. 889, 895, 900; Moscati 2005, 216, n. 67).

57 Taloni 2012b, con bibliografia precedente.

58 Amon è la divinità principale del pantheon egizio durante il Nuovo Regno sotto la XVII, XIX e XX Dinastia. È il dio creatore associato a Ra.

59 La raffigurazione dell'ariete non necessariamente presuppone che questo venisse offerto in sacrificio nel Tempio. Al contrario, i reperti ossei e l'assenza di crani e corna di ariete inducono a pensare che questo non fosse incluso tra gli animali solitamente sacrificati (una situazione analoga si riscontra a Creta nel secondo millennio a.C. [Hallager 2001, 319]). In ambito miceneo e greco, al contrario gli arieti erano sacrificati alle principali divinità olimpie e anche quelle prettamente ctonie. Si ricordi in particolare il sacrificio di un ariete da parte di Odisseo alle porte dell'Ade dove, grazie al sangue dell'animale sacrificato, di cui si nutrivano i morti, era potuto entrare in contatto con le anime dei defunti (Odissea 10:521-537; 11:24-37). In questo caso la scelta dell'ariete come vittima sacrificale è connessa al valore simbolico di rigenerazione e fertilità attribuito all'animale.

60 A margine del discorso è possibile avanzare anche un'altra ipotesi interpretativa che non è in contrasto con la precedente ma che, in un cero senso, contribuisce a delineare il contesto cultuale di riferimento. La brocchetta con protome d'ariete, infatti, può essere legata alla divinità femminile venerata nell'area sacra, Astarte, paredra di Baal e titolare dell'edificio sacro posto immediatamente a nord del tempio maggiore. La Astarte dell'Area Sacra del Kothon manifesta, proprio a partire dai primi decenni del V secolo a.C., un forte carattere ctonio a fianco di quello astrale e celeste (Nigro 2015, 240, fig. 17). Questo si concretizza nel sincretismo con Demetra, particolarmente evidente in particolari contesti cultuali di V secolo a.C. come i pozzi sacri del settore sud occidentale dell'area (Nigro - Spagnoli 2012; Spagnoli 2013). Come simbolo di fertilità e di rigenerazione l'ariete, quindi, potrebbe essere un riferimento alla Malophoros, "Colei che porta frutti” o “Colei che porta greggi” (Pausania I 44,3). 


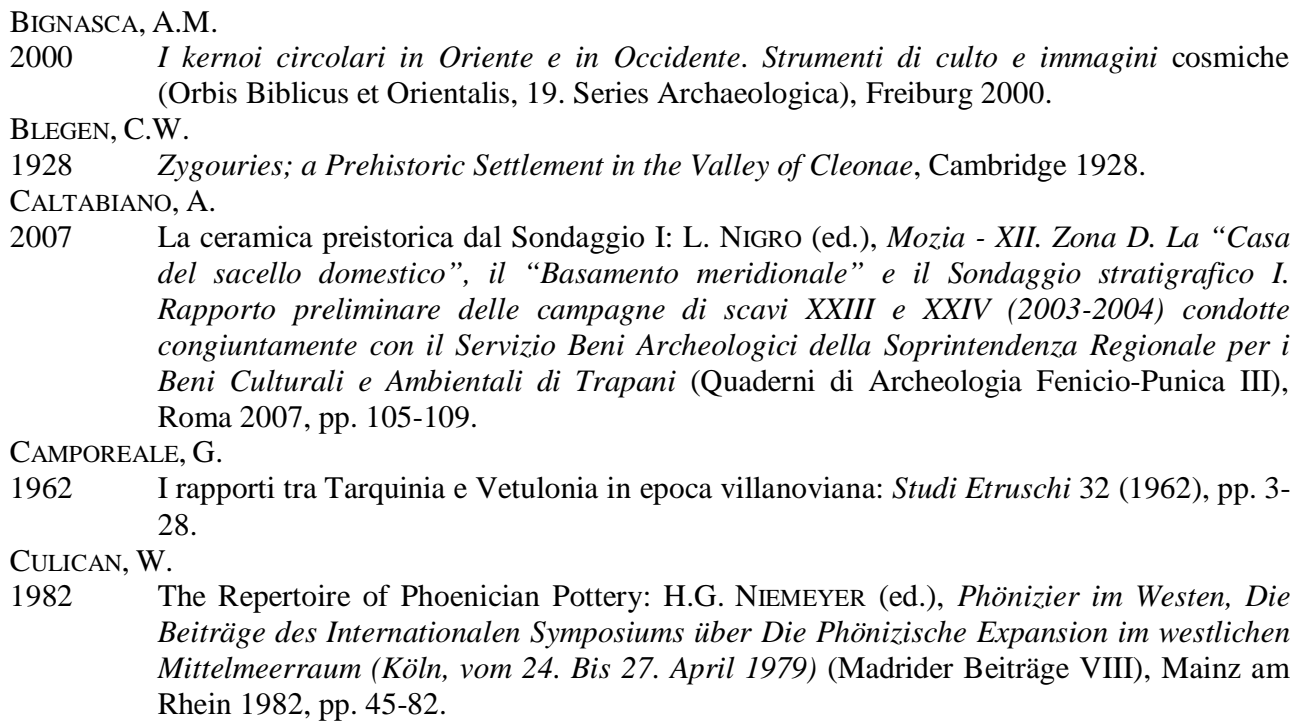

2007 La ceramica preistorica dal Sondaggio I: L. Nigro (ed.), Mozia - XII. Zona D. La "Casa del sacello domestico", il "Basamento meridionale" e il Sondaggio stratigrafico I. Rapporto preliminare delle campagne di scavi XXIII e XXIV (2003-2004) condotte congiuntamente con il Servizio Beni Archeologici della Soprintendenza Regionale per i Beni Culturali e Ambientali di Trapani (Quaderni di Archeologia Fenicio-Punica III), Roma 2007, pp. 105-109.

CAMPOREALE, G.

1962 I rapporti tra Tarquinia e Vetulonia in epoca villanoviana: Studi Etruschi 32 (1962), pp. 328.

CULICAN, W.

1982 The Repertoire of Phoenician Pottery: H.G. NiemeYer (ed.), Phönizier im Westen, Die Beiträge des Internationalen Symposiums über Die Phönizische Expansion im westlichen Mittelmeerraum (Köln, vom 24. Bis 27. April 1979) (Madrider Beiträge VIII), Mainz am Rhein 1982, pp. 45-82. CVA Oxford 2

BeAZley, J.D. - PAyne, H.G.G. - PRice, E.R.

Corpus Vasorum Antiquorum, Great Britain, Fascicule 9. Oxford Ashmolean Museum, Fascicule 2, London - Paris 1931.

CVA Copenhagen 2

BlinKenBerg, Chr. - Fris JoHANSEN, K.

Corpus Vasorum Antiquorum Danemark. Copenague 2: Musée National (Collection des D’ANDREA, B. Antiquités Classiques), Paris 1927.

2014 I Tofet del Nord Africa dall'età arcaica all'età romana (VIII sec. a.C.- II sec. d.C.). Studi archeologici (Collezione di Studi fenici 45), Pisa - Roma 2014.

DOTHAN, M. - PORATH, Y.

1982 Ashdod - IV. Excavation of Area M, Jerusalem 1982.

DOUMET-SERHAL, C.

2001 A Ram's Head Handle from Sidon: Archaeology and History in Lebanon Issue Thirteen, Spring 2001, pp. 9-16.

EPSTEIN, C.

1985 Laden Animal Figurines from the Chalcolithic Period in Palestine: Bulletin of the American School of Oriental Research 258 (1985), pp. 53-62.

GRAN AYMERICH, J.M.J.

1983 La céramique phénico-punique et le bucchero étrusque: cas concrets et considérations générales: P. BARTOLONI - S.F. Bondì - G. COACCI POLSELLI - M.T. FrANCISI - F. MAZZA G. Petruccioli - P. Xella (a cura di), Atti del I Congresso Internazionale di studi fenici e punici (Roma 5-10 novembre 1979), Roma 1983, pp. 77-87.

GRAU-ZIMMERMAN, B.

1978 Phönikische Metallkannen in den orientalisierenden Horizonten des Mittelmeerraumes: Madrider Mitteilungen 19 (1978), pp. 161-218. 
GuIZZI, F.

2012 Graffito con dedica votiva su un aryballos dall'area sacra del Kothon a Mozia: L. NigRo F. Spagnoli, Alle sorgenti del Kothon. Il rito a Mozia nell'Area sacra di Baal 'Addir Poseidon. Lo scavo dei pozzi sacri nel Settore C Sud-Ovest (2006-2011) (Quaderni di Archeologia Fenicio-Punica/Colour Monograph 02), Roma 2012, pp. 13-15. JiMÉNEZ ÁVILA, J.

2002 La toréutica orientalizante en la Península Ibérica (Bibliotheca Archaeologica Hispana 16), Madrid 2002.

HALLAGER, B.P.

2001 The Ram in Cultic Contexts?: Aegeum 22 (2001), pp. 315-320.

HEMINGWAY, S.

2013 The Metropolitan Museum Art Bulletin, Spring 2012, vol. 69/4, New York 2013.

HENCKEN, $\mathrm{H}$.

1968 Tarquinia, Villanovians and Early Etruscan, Cambridge 1968.

KARAGEORGHIS, V.

1977 Two Cypriote Sanctuaries of the End of the Cypro-Archaic Period, Rome 1977.

I Fenici

1988 S. Moscati (a cura di), I Fenici. Catalogo della mostra, Palazzo Grassi, Venezia, 6 marzo - 6 novembre 1988, Milano 1988.

LANCEL, S.

$1992 \quad$ Carthage, Paris 1992.

MiNTO, A.

1934 Le ultime scoperte archeologiche di Populonia (1927-1931): Monumenti Antichi dei Lincei 34 (1934), pp. 362-378.

Moscati, S.

2005 Fenici e Cartaginesi in Sardegna (Biblioteca Sarda, n. 102). Seconda edizione a cura di P. Bartoloni, Nuoro 2005.

NeEFT, C.W.

1987 Protocorinthian Subgeometric Aryballoi, Amsterdam 1987.

NigRO, L.

2009 Offerte e depositi votivi nel Santuario C3 del Kothon di Mozia nel IV secolo a.C.: S. ForTUNELli - C. MASSERIA (a cura di), Ceramica attica da santuari della Grecia, della Ionia e dell'Italia (Atti Convegno Internazionale Perugia 14-17 marzo 2007), Venosa 2009, pp. 703-719.

2010 Alle origini di Mozia: stratigrafia e ceramica del Tempio del Kothon dall'VIII al VI secolo a.C.: L. Nigro (ed.), Motya and the Phoenician Ceramic Repertoire between the Levant and the West, 9th-6th Century BC. Proceedings of the International Conference held in Rome, $26^{\text {th }}$ February 2010 (Quaderni di Archeologia Fenicio-Punica, V), Rome 2010, pp. 1-48.

2013a Il Tofet e la città. Il limite meridionale del Santuario e le strutture collegate negli scavi della Sapienza 2010-2011: Scienze dell'Antichità 19/1 (2013), pp. 37-53.

2013b Before the Greeks: The earliest Phoenician settlement in Motya - Recent discoveries by Rome “La Sapienza” Expedition: Vicino Oriente 17 (2013), pp. 39-74.

2015 Mozia tra VI e V secolo a.C. Monumentalizzazione e organizzazione socio-politica: un nuovo modello: M.P. BAGLIONE - L.M. MichetTi (a cura di), Le lamine d'oro a cinquant'anni dalla scoperta. Dati archeologici su Pyrgi e rapporti con altre realtà del Mediterraneo (Scienze dell'Antichità 21.2), Roma 2015, pp. 225-245. 
NigRo, L. (ed.)

2004 Mozia - X. Zona C. Il Kothon. Zona D. Le pendici occidentali dell'Acropoli. Zona F. La porta ovest. Rapporto preliminare della XXII campagna di scavi - 2002 condotta congiuntamente con il Servizio Beni Archeologici della Soprintendenza Regionale per $i$ Beni Culturali e Ambientali di Trapani (Quaderni di Archeologia Fenicio-Punica, I), Roma 2004

2005 Mozia - XI. Il Tempio del Kothon. Rapporto preliminare delle campagne di scavi XXIII e XXIV (2003-2004) condotte congiuntamente con il Servizio Beni Archeologici della Soprintendenza Regionale per i Beni Culturali e Ambientali di Trapani (Quaderni di Archeologia Fenicio-Punica, II), Roma 2005.

NigRo, L. - SPAGNOLI, F.

2012 Alle sorgenti del Kothon. Il rito a Mozia nell'Area sacra di Baal 'Addir - Poseidon. Lo scavo dei pozzi sacri nel Settore C Sud-Ovest (2006-2011) (Quaderni di Archeologia Fenicio-Punica/Colour Monograph 02), Roma 2012.

NUÑEZ, F.J.

2004 Preliminary report on ceramics from the Phoenician necrópolis of Tyre - Al-Bass. 1997 campaign: M.E. AuBET, The Phoenician Cemetery of Tyre-al Baas. Excavations 19971999 (Bulletin d’Archéologie et d’Architecture Libanaises Hors-Série I), Beirut 2004, pp. 281-373.

Paris 2007

2007 «La Méditerranée des Phéniciens: de Tyr à Carthage». Catalogue de l'exposition à l'Institute du Monde Arabe, Paris, 6 novembre 2007-20 avril 2008, Paris 2007. SPAGNOLI, F.

2007 La ceramica comune punica dallo strato di pareggiamento US.1111 e dal riempimento della fossa F.1112b: il repertorio del VII-VI secolo a.C.: L. NigRo (ed.), Mozia - XII. Zona D. La "Casa del sacello domestico", il "Basamento meridionale” e il Sondaggio stratigrafico I. Rapporto preliminare delle campagne di scavi XXIII e XXIV (2003-2004) condotte congiuntamente con il Servizio Beni Archeologici della Soprintendenza Regionale per i Beni Culturali e Ambientali di Trapani (Quaderni di Archeologia FenicioPunica III), Roma 2007, pp. 93-102.

2013 Demetra a Mozia: evidenze dall'area sacra del Kothon nel V secolo a.C.: Vicino Oriente 17 (2013), pp. 153-165.

2014 Una brocchetta dipinta dal Tempio di Astarte nell’Area Sacra del Kothon a Mozia: Vicino Oriente 18 (2014), pp. 113-121.

2015 In the nostrils of God: stone incense altars in Phoenician cult contexts: A.M. MAILAAfEICHE (ed.), Cult and Ritual on the Levantine Coast and its impact on the Eastern Mediterranean Realm. Proceedings of the International Symposium, Beirut 2012 (Bulletin SPARKS, R.T. d’Archéologie et d'Architecture Libanaises, Hors-Série X), Beyrouth 2015, pp. 215-234.

1991 A Series of Middle Bronze Age Bowls with Ram’s-head Handles from the Jordan Valley: Mediterranean Archaeology 4 (1991), pp. 45-54.

SPARKES, B.A. - TALCOTT, L.

1970 Athenian Agora, XII. Black and Plain Pottery of the $6^{\text {th }}, 5^{\text {th }}$ and $4^{\text {th }}$ Centuries B.C., Princeton 1970.

STOOP, M.W.

1976 Francavilla Marittima. Acropoli sulla Motta: Atti e Memorie della Società Magna Grecia, notizie storiche 15-17 (1974-1976), pp. 107-167. 
TALONI, M.

2012a Ram-Headed oinochoai: M.C. Biella - E. Giovanelli - L.G. Perego (a cura di), Il Bestiario fantastico di età orientalizzante nella Penisola Italiana (Aristonothos. Scritti per il Mediterraneo antico), Milano 2012, pp. 371-397.

2012b Le oinochoai cosiddette fenicio-cipriote: origine, rielaborazione e trasformazione di una forma vascolare: C. REgoli (ed.), Mode e Modelli. Fortuna e insuccesso nella circolazione di cose e idee (Officina Etruscologia 7), Roma 2012, pp. 77-98.

TUSA, V.

1964 Il Cappiddazzu. Lo scavo del 1964: A. CiASCA - M. ForTE - G. GARBINI - S. MosCATI - B. PUGLIESE - V. TUSA, Mozia - I. Rapporto preliminare della Missione archeologica della Soprintendenza alle Antichità della Sicilia Occidentale e dell'Università di Roma (Studi Semitici 12), Roma 1964, pp. 33-40.

1972 La Necropoli Arcaica e adiacenze. Lo scavo del 1970: F. BevilacQua - A. Ciasca- G. Matthiae Scandone- S. Moscati - V. TuSA - A. Tusa Cutroni, Mozia - VII. Rapporto preliminare della Missione congiunta con la Soprintendenza alle Antichità della Sicilia Occidentale (Studi Semitici 40), Roma 1972, pp. 7-81.

XeLla, P.

1991 Baal Hammon: recherches sul l'identité et l'histoire d'un dieu phénico-punique (Collezione di studi fenici 32), Roma 1991.

VERMEULE, C.

1974 The Ram Cults of Cyprus. Pastoral to Paphian at Morphou: Report of the Department of Antiquities of Cyprus (1974), pp. 151-156. 

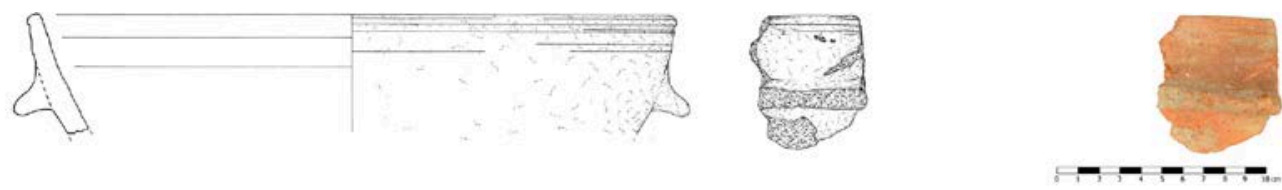

MC.15.5108/35

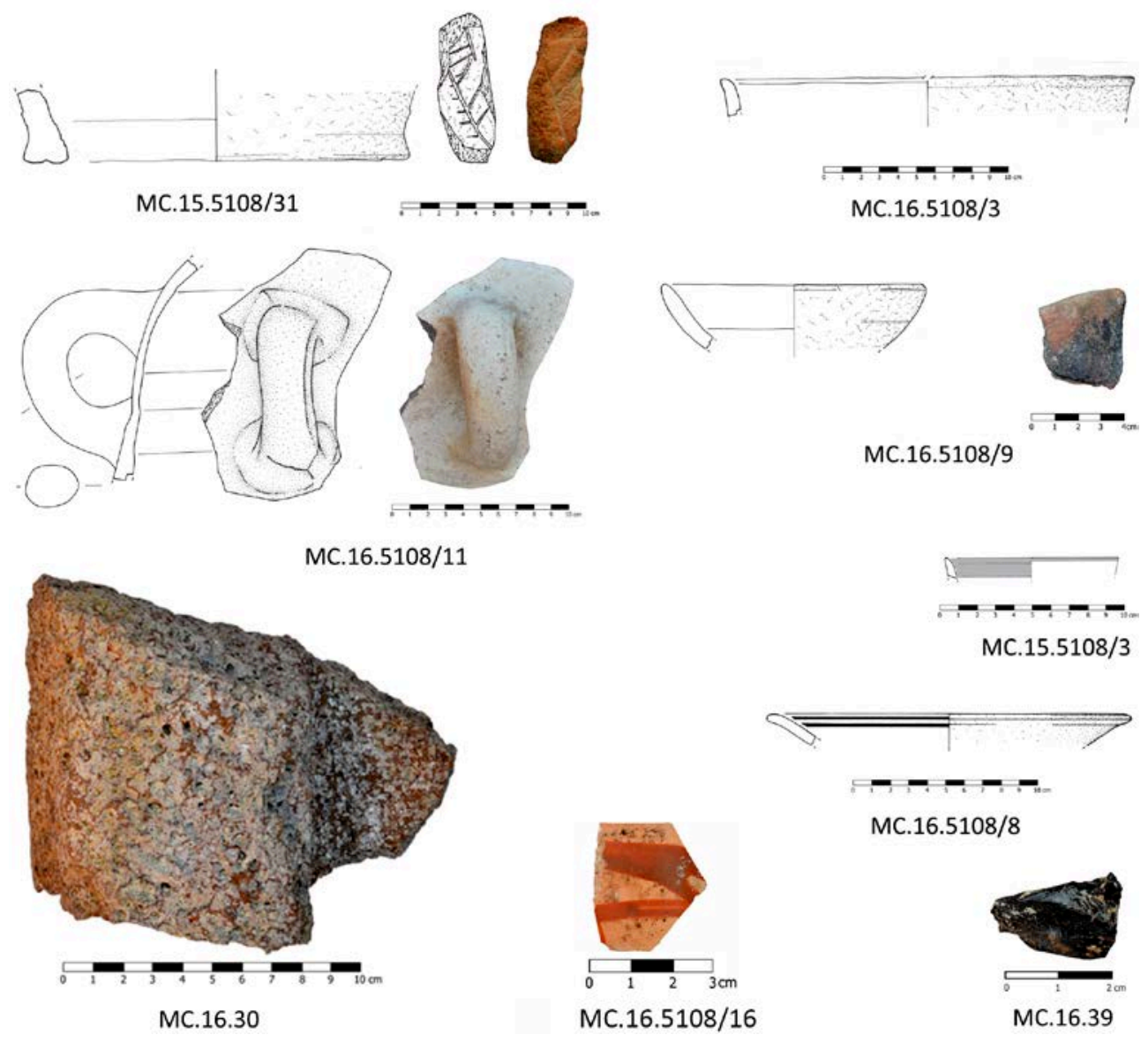

Fig. 1 - I materiali di VIII - primo quarto VII secolo a.C. (Motya IVA, 800-750 a.C.; Motya IVB, 750-675 a.C.) rinvenuti nella colmata US.5108. 


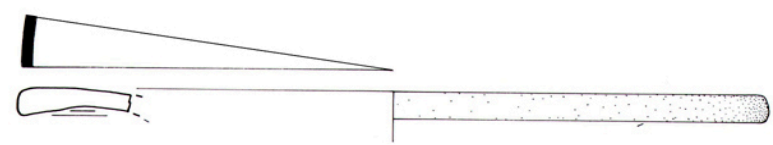

MC.15.5108/15

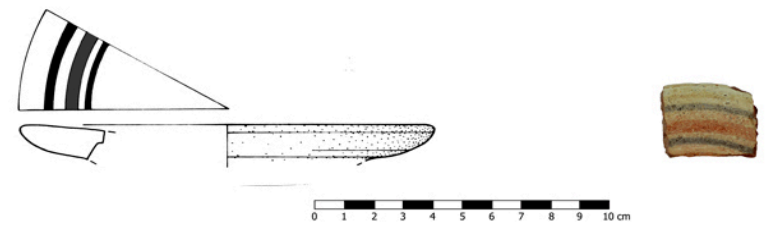

MC.16.5108/6

Fig. 2 - La ceramica di VII secolo a.C. (Motya VA, 675-625 a.C.) rinvenuta nella colmata US.5108.
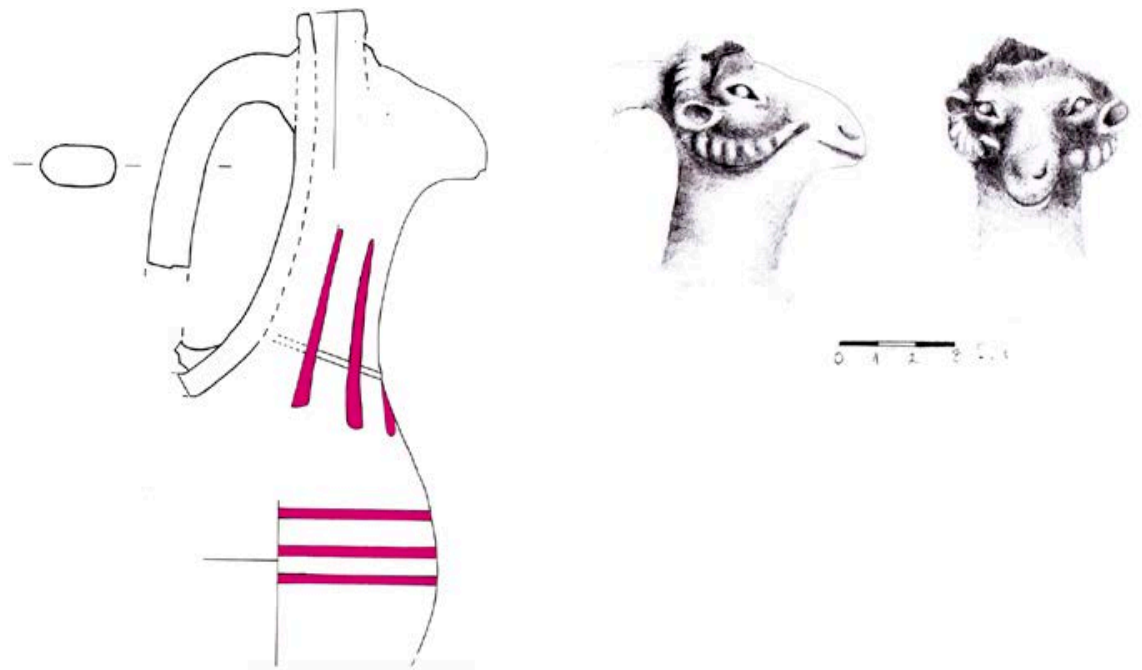

Fig. 3 - La brocchetta MC.15.5108/1, sezione del vaso, profilo e prospetto della protome d'ariete (disegno A. Sumislawska). 

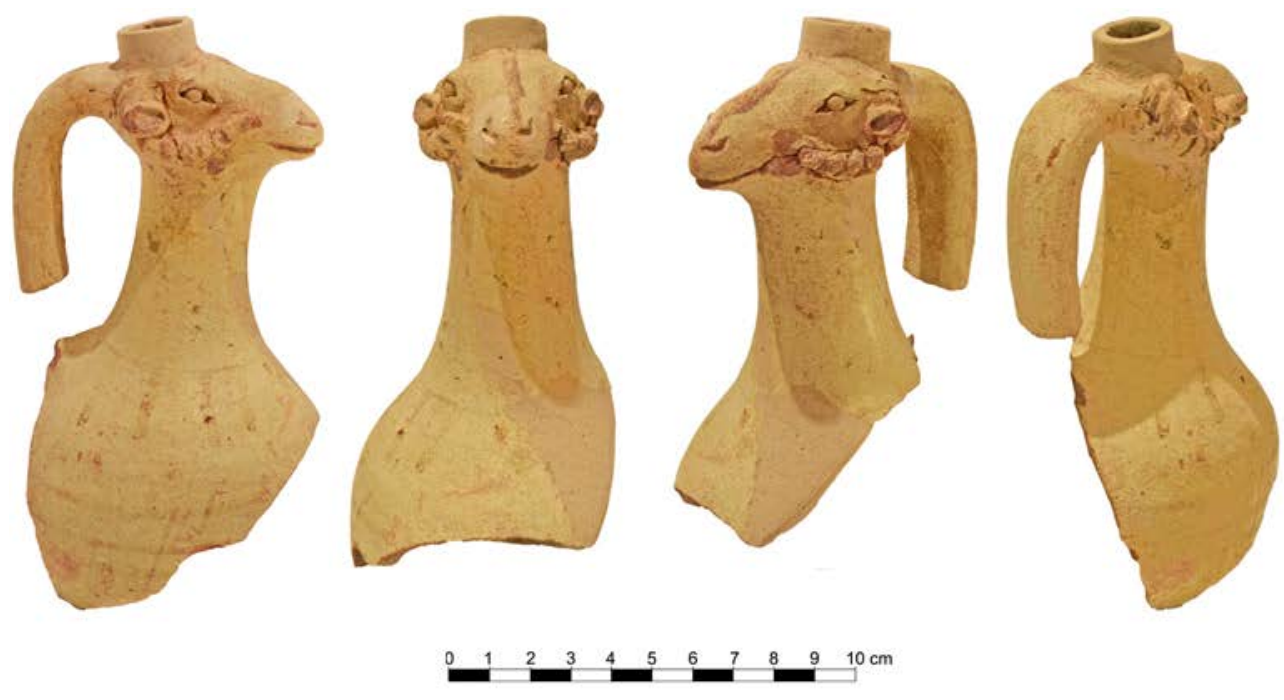

\section{MC.15.5108/1}
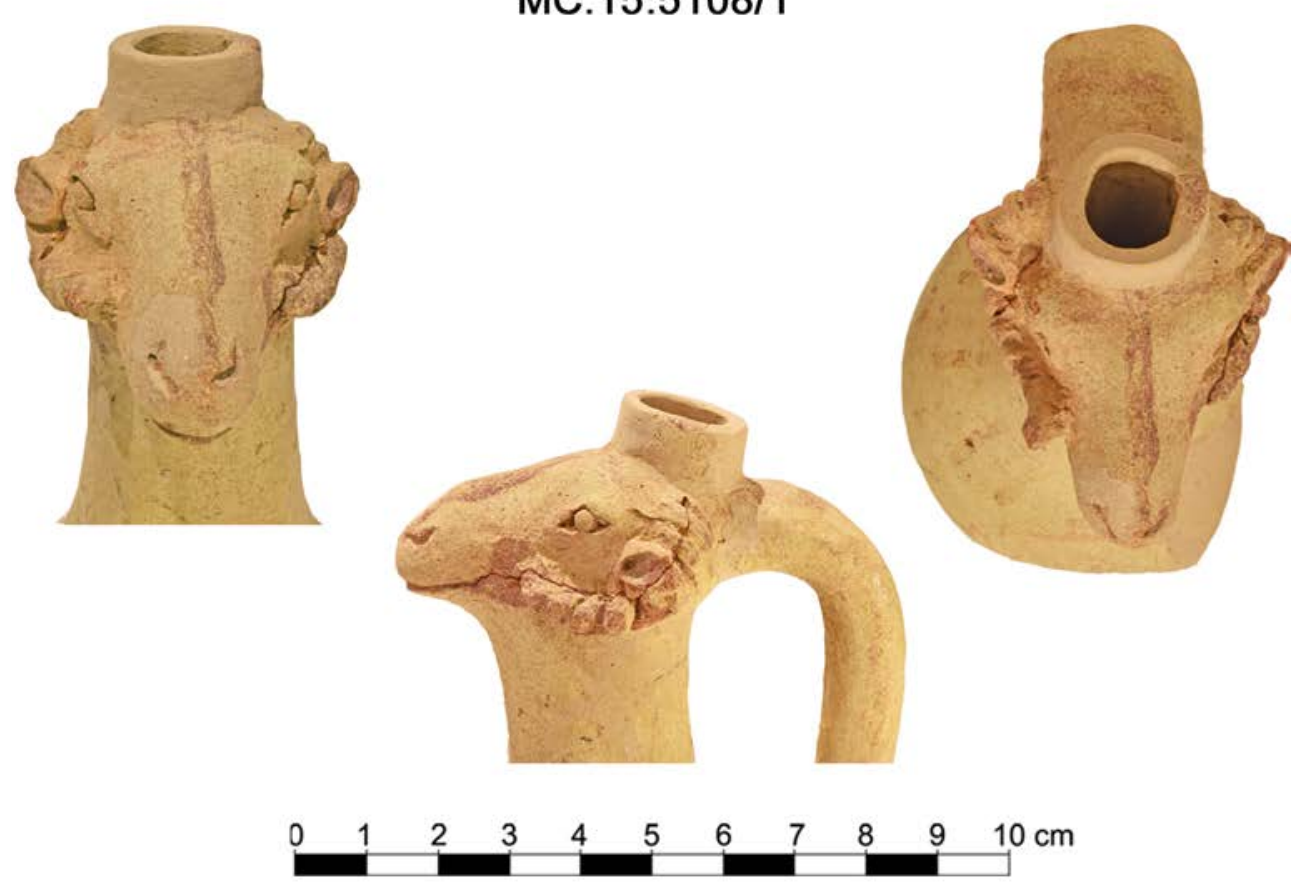

Fig. 4 - La brocchetta MC.15.5108/1, decorazione dipinta. 


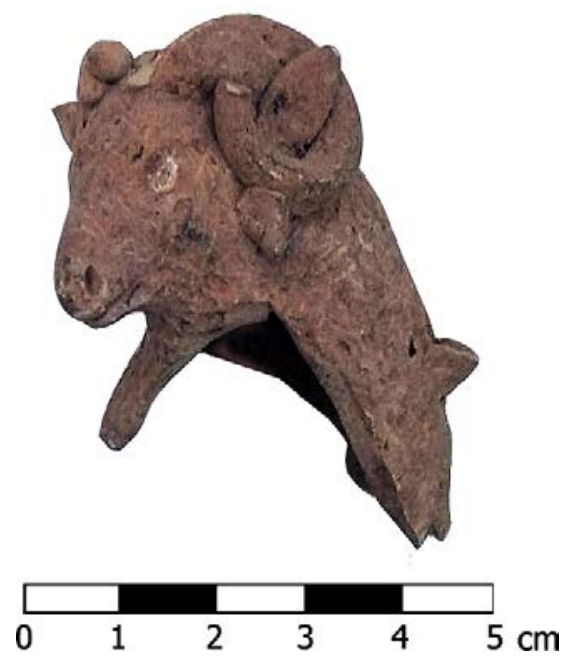

Fig. 5 - La stautetta di ariete rinvenuta nell'area del Cappiddazzu, V secolo a.C. (Foto Museo G. Withaker).

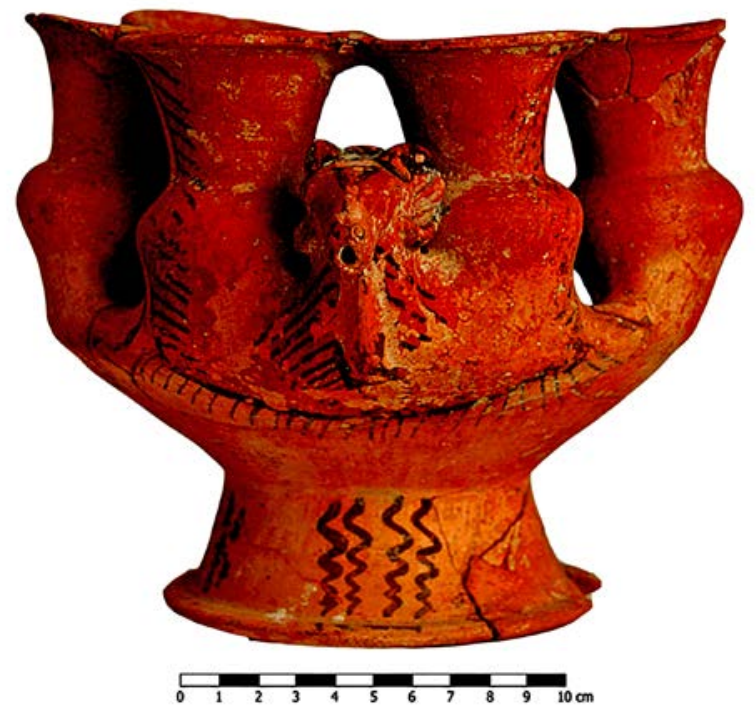

Fig. 6 - Il kernos con protome d'ariete dalla Tomba 29 della Necropoli Arcaica (Foto Museo G. Withaker). 